\title{
The Abuse Characteristics of Amphetamine-Type Stimulants in Patients Receiving Methadone Maintenance Treatment and Buprenorphine Maintenance Treatment
}

\author{
Yue Liu' \\ Nina Liu ${ }^{2}$ \\ Wenwen Shen (iD) \\ Longhui $\mathrm{Li}^{1}$ \\ Wenhua Zhou' \\ Leiting $\mathrm{Xu}^{3}$ \\ 'Department of Academic Research, \\ Ningbo Kangning Hospital, Key \\ Laboratory of Addiction Research of \\ Zhejiang Province, Ningbo, 31520I, \\ People's Republic of China; ${ }^{2}$ Department \\ of Basic Medicine, Ningbo College of \\ Health Sciences, Ningbo, 315100, \\ People's Republic of China; ${ }^{3}$ Department \\ of Basic Medicine, Ningbo University \\ School of Medicine, Ningbo, 3152II, \\ People's Republic of China
}

Correspondence: Leiting $\mathrm{Xu}$

Department of Basic Medicine, Ningbo University School of Medicine, Ningbo,

3152II, People's Republic of China

Tel +86 I82674I7969

Email leitingxu@yeah.net

Wenhua Zhou

Department of Academic Research, Ningbo Kangning Hospital, Key

Laboratory of Addiction Research of

Zhejiang Province, Ningbo, 31520I,

People's Republic of China

Tel +86 I3777II 3984

Email wenhuazhou@yeah.net
Objective: The purpose of this study was to retrospectively investigate the abuse characteristics of amphetamine-type stimulants (ATS) in patients receiving methadone maintenance treatment (MMT) and buprenorphine maintenance treatment (BMT).

Methods: A total of $58 \mathrm{MMT}$ and $51 \mathrm{BMT}$ patients abusing ATS were recruited from the drug maintenance treatment clinic of Ningbo Addiction Research and Treatment Center from January 2018 to December 2019. They were assessed using the amphetamine abuse questionnaire (AAQ), addiction severity index (ASI) and Barratt impulsiveness scale (BIS). Moreover, 40 MMT control patients, 40 BMT control patients and 20 healthy controls were also assessed using the BIS. All information was collected using the amphetamine abuse questionnaire (AAQ), Chinese version of addiction severity index (ASI-C) and Chinese version of Barratt impulsiveness scale (BIS-C) conducted by qualified psychologists.

Results: The interval of amphetamine use in the MMT group was shorter than the BMT group $(P<0.05)$. The drug use subscale score of ASI was higher in the MMT group than the BMT group $(P<0.05)$. The respective and total scores of attentional impulsiveness, motor impulsiveness and non-planning impulsiveness in BIS in the MMT group were all higher than the MMT control group $(P<0.05)$. The scores of motor impulsiveness and nonplanning impulsiveness in the BMT group were higher than the BMT control group $(P<$ 0.05). The respective and total scores in BIS in the MMT control group and the BMT control group were all higher than those in the healthy controls.

Conclusion: The patients showing amphetamine abuse in maintenance therapy had a greater impulsiveness than those having other simple maintenance treatments, and patients under MMT may be more addicted to amphetamines in comparison with those having BMT.

Keywords: methadone maintenance treatment, buprenorphine maintenance treatment, amphetamine-type stimulants, impulsiveness, addiction

\section{Introduction}

Opioid use disorder (OUD) is a significant public health problem with excessive morbidity and mortality, as well as increasing criminality. ${ }^{1}$ Tsui et al. in 2018 reported that OUD is closely intertwined with injection drug use, which is a major driver of chronic viral infections, such as hepatitis $\mathrm{C}$ virus (HCV) and human immunodeficiency virus (HIV). ${ }^{2}$ Opioid maintenance therapy (OMT), as a drug substitution therapy, is one of the most widely accepted methods for the treatment of OUD. ${ }^{3}$ OMT is reported to play 
an important role in reducing criminality in opioid addicts, preventing the relapse of heroin addicts and controlling the spread of AIDS and other infectious diseases. ${ }^{4}$ Synthetic cathinone use among opiate-dependent patients is still a current phenomenon in contemporary addiction care. ${ }^{5}$ Among the available OMTs, methadone maintenance treatment (MMT) and buprenorphine maintenance treatment (BMT) are two well-recognized effective replacement therapies for opiate dependence.

Since 2004, community-based MMT has been widely used in China as an effective strategy to reduce the harm of drug abuse and prevent AIDS. Nowadays, MMT clinics in China have covered almost all areas where opioid addicts are concentrated. ${ }^{6}$ Buprenorphine has the advantages of convenient carrying, convenient use, no need to take it regularly in the outpatient clinic, and unlimited outdoor activities for patients. ${ }^{7}$ Therefore, it is widely accepted by some addicts. BMT has been carried out in many drug maintenance treatments in China to provide individualized medical guidance for OUD patients in clinics. However, long-term follow-up studies have showed that poor compliance and high rates of dropout and relapse were problematic for both MMT and BMT, which results in inefficient use of limited resources and unsatisfactory abstinence attainment rate. ${ }^{8,9} \mathrm{~A}$ previous study has reported that in some outpatient clinics, the number of people receiving drug maintenance treatment had even dropped to $40 \%$ or less five years ago. ${ }^{10}$

Multi-drug abuse may involve new types of drugs. Amphetamine type stimulants (ATS) are one of the most important synthetic drugs controlled by the United Nations Convention on psychotropic substances. ATS have a central nervous excitatory effect, which exerts influence on the reward system of the human brain, resulting in dependence and abuse. As the United Nations Office on Drugs and Crime reported, abuse of the illegal psychostimulant, methamphetamine (METH), has become an international public health problem with an estimated 15-16 million users worldwide, a total which exceeds the number of people who abuse heroin and cocaine and makes METH the second most widely abused drug after cannabis. ${ }^{11}$

Owing to their stimulating psychological effects, ATS are mainly associated with the club scene. Together with environmental factors such as hyperthermia and dehydration, ATS are assumed to exhibit neurotoxic properties even in recreational users. ${ }^{12}$
ATS abuse not only causes serious social problems, but also brings serious damage to the physical and mental health of abusers. ATS can cause serious neuropsychiatric damage to the brain, which can produce irreversible neuropsychiatric disorders. Compared with opioids, the withdrawal symptoms of ATS dependence are mainly manifested as strong drug craving, emotional fluctuations, anxiety, insomnia, and suicidal intention. ATS are the major factor that affects treatment adherence and therapeutic efficacy of OMT. ${ }^{13-15}$ The reason is that OMT is mainly aimed at patients with opioid addiction, which is a chronic and recurrent brain disease. It also leaves the patients at high risk of new drug abuse. ${ }^{16}$ Therefore, multidrug abusers present overlapping characteristics of both traditional and new drug abusers. They have characteristics different from those of the users only using new type drugs, including older age, lower income, intermittent use of new drugs, and more diseases such as HIV infection and hepatitis $C .{ }^{17,18}$

Accumulating evidence suggests that impulsivity may be a behavioral marker of the propensity to take addictive drugs. ${ }^{19-21}$ Impulsivity is significantly higher in the addicts to opioid, cocaine or other drugs than in healthy controls. $^{22-26}$ The higher the trait impulse, the shorter the duration of drug maintenance treatment. ${ }^{27,28}$ In addition, studies have shown that there is a positive association between impulsivity and cocaine use severity indicated by average daily cocaine use, craving and cocaine withdrawal symptoms. ${ }^{29}$ However, it is not clear whether MMT and BMT patients using ATS have different impulsivity and addiction severity as previous opioid addicts. Therefore, the purpose of this study was to retrospectively investigate the abuse characteristics of amphetamine-type stimulants (ATS) in patients receiving methadone maintenance treatment (MMT) and buprenorphine maintenance treatment (BMT).

\section{Materials and Methods}

\section{Participants}

This study was approved by the ethics committee of Ningbo Institute of Microcirculation and Henbane and performed in accordance with the Declaration of Helsinki, and all participants signed written informed consent. Ethics trial no. is 330201013-201900004. Participants were recruited from the drug maintenance treatment clinic of of Ningbo Addiction Research and Treatment Center from January 2018 to December 2019. Inclusion criteria: (1) 
patients who were diagnosed as having amphetamine addiction according to the ICD-11 Diagnostic Criteria for Amphetamine Dependence; (2) patients with age between 18 and 55 years; (3) patients without serious physical/psychiatric illness; (4) patients without verbal communication barrier; (5) patients who showed a longer history of AST abuse. Exclusion criteria: (1) patients who began to abuse AST before entering the drug maintenance therapy or after entering the drug maintenance therapy; (2) patients having severe physical/mental disorders, mental disability or speech disorders; (3) females with a positive pregnancy test.

There were 58 patients currently receiving MMT and 51 receiving BMT. In the impulsivity study, participants in the MMT control $(\mathrm{n}=40)$ and BMT control $(\mathrm{n}=40)$ groups were under MMT or BMT, respectively, and they did not abuse ATS. They were recruited from patients in the clinic of drug maintenance therapy. All those participants provided valid questionnaires.

\section{Medication}

In this study, patients in the MMT and BMT groups (MMT group, BMT group, MMT control group and BMT control group) had received maintenance therapy for more than 24 months. Their maintenance dose had been maintained at a stable level after the initial induction period. The lowest daily maintenance dose of methadone was $10 \mathrm{mg}$ and the highest was $120 \mathrm{mg}$, with an average daily dose of $60-80 \mathrm{mg}$. The lowest daily maintenance dose of buprenorphine was $0.5 \mathrm{mg}$ and the highest was $5.0 \mathrm{mg}$, with an average daily dose of 3-4.5 mg. The determination of dosage was based on the principle of dosage individuation, the ideal control of withdrawal symptoms and drug craving, subjective acceptability, and no impact on consciousness and occupational function of the patients.

\section{Measurements}

All information were collected using the amphetamine abuse questionnaire (AAQ), Chinese version of addiction severity index (ASI-C) and Chinese version of Barratt impulsiveness scale (BIS-C) conducted by qualified psychologists.

\section{Amphetamine Abuse Questionnaire (AAQ)}

We use open questions in the AAQ to explore potential reasons for ATS use. The AAQ is actually a record of an interview between a doctor and a subject. We set up a number of related questions in it. The AAQ covers demographic information and drug-use related questions. Demographic information includes gender, age, marital status, occupation, and years of education. The drug-use related questions comprise length, interval and pattern of drug abuse, age and reason of first use, opioid use in the last 30 days, and current comorbidities.

\section{Chinese Version of Addiction Severity Index (ASI-C)}

The ASI is a standard structured questionnaire for drug abusing and dependent patients, and it is applicable to the evaluation of multiple drug use problems. ${ }^{21,30}$ It is composed of 7 subscales, reflecting physical health, occupational/social support, drug use, alcohol abuse, crime, family/social relations, and mental status, respectively. The 7 subscales have 7 composite scores, ranging from 0 to 1 . The closer score is to 1 , the more serious the problems are in respective domains. On the contrary, the closer score is to 0 , which shows that the addict has fewer problems. The ASI was translated into Chinese and evaluated by Liang et al. It shows ASI-C in drug users had enough good construct and discriminant validity in China. ${ }^{31}$

\section{Chinese Version of Barratt Impulsiveness Scale (BIS-C)}

The BIS is a self-report measure for assessing individual impulsive personality traits. Since being developed by Barratt, BIS has gone through 15 versions, but the version BIS-11 revised by Patton is the most commonly used. ${ }^{32}$ BIS-11 was translated and revised by Zhou et al. in China. ${ }^{33}$ BIS-11-C shows good reliability and validity, which meets the needs of psychometric properties, and can be used in the research of impulsivity in the social cultural context of China. BIS-11-C has 30 items and a three factor super structure that taps important dimensions of trait impulsivity, including attentional impulsiveness, non-planning impulsiveness and motor impulsiveness. ${ }^{34}$ Each participant was asked to answer questions that measured their behavior or way of thinking. They were also asked to rate the extent of the stated questions. Each subscale uses a 4-point scale: "never" is rated 1, "occasionally" is 2, "often" is 3 and "always" is 4 . Subscale scores can be combined into a total score ranging 
from 30 to 120 . The higher a participant's overall score, the greater impulsivity they have.

\section{Data Analysis}

All data were processed by SPSS 24.0 statistical software. An independent sample $t$-test was used for comparing continuous variables while the chi-square test was used for categorical data. $P<0.05$ was considered statistically significant. One-way ANOVA followed by Bonferroni post hoc tests to compare all pairs of groups was performed to assess the differences between multiple groups. A $P$ value $<0.05$ was considered as statistically significant.

\section{Results}

\section{Demographic Characteristics}

A total of 130 patients were invited; 118 participants provided questionnaires and underwent urine toxicology tests of morphine and amphetamines. Nine were further excluded due to missing, false and incomplete answers in questionnaires. Therefore, 109 participants were finally included in the current analysis. Patients in the MMT group and MMT control group were matched in gender, age, marriage, years of education and other aspects, as well as patients in the BMT group and BMT control group. $71 \%$ of the participants were male. There were no significant differences in age and education level between MMT group and MMT control group, as well as BMT group and BMT control group $(P>0.05) .46 .8 \%$ of the participants were unmarried or divorced and $30.2 \%$ were unemployed (Table 1).

\section{Amphetamine Use Characteristics}

No significant differences were found in the mean duration of amphetamine use, age of first drug use, duration of drug maintenance treatment, presence of comorbidity of mental disorders, HIV infection and sleep disorders $(P>0.05)$. However, compared with the MMT group, patients in the BMT group had significantly longer drug abuse interval ( $(9.15 \pm 5.51)$ days vs $(5.53 \pm 3.99)$ days; $P=0.039)$, fewer years of opioid abuse before maintenance treatment $((3.34 \pm 3.53)$ years vs $(7.42 \pm 6.41)$ years; $P=0.046)$ and smaller daily dose of opioid use $((1.68 \pm 0.67) \mathrm{g}$ vs $(1.17 \pm 0.68) \mathrm{g} ; P=0.041)$ (Table 2$)$. Furthermore, the main reasons for the first use of the new drug in $53.2 \%$ of the participants were curiosity and the influence of friends. Other reasons included self-
Table I Demographic Characteristics

\begin{tabular}{|c|c|c|c|}
\hline Characteristics & $\begin{array}{l}\text { MMT } \\
(n=58)\end{array}$ & $\begin{array}{l}\text { BMT } \\
(n=5 I)\end{array}$ & $P$-value \\
\hline Age (years) & $35.00 \pm 7.06$ & $31.77 \pm 5.17$ & 0.169 \\
\hline Gender (n,\%) & & & 0.406 \\
\hline Male & 39 (67.2\%) & 38 (74.5\%) & \\
\hline Female & $19(32.8 \%)$ & $13(25.5 \%)$ & \\
\hline Marital status (n,\%) & & & 0.985 \\
\hline Married & 31 (53.4\%) & 27 (52.9\%) & \\
\hline Unmarried & 14 (24.1\%) & I 3 (25.4\%) & \\
\hline Divorced & 13 (22.4\%) & II (2I.6\%) & \\
\hline Occupation (n,\%) & & & 0.077 \\
\hline Full-time & $12((20.7 \%))$ & $20(39.2 \%)$ & \\
\hline Part-time & $28(48.3 \%)$ & $16(31.4 \%)$ & \\
\hline Unemployed & 18 (3I.0\%) & 15 (29.4\%) & \\
\hline Years of education (years) & $9.42 \pm 1.70$ & $10.15 \pm 1.95$ & 0.269 \\
\hline Family relation (n,\%) & & & 0.783 \\
\hline Harmonious & $13(22.4 \%)$ & $13(25.5 \%)$ & \\
\hline Inharmonious & 17 (29.3\%) & $12(23.5 \%)$ & \\
\hline General & $28(48.3 \%)$ & $26(51.0 \%)$ & \\
\hline Medical conditions $(\mathrm{n}, \%)$ & & & 0.480 \\
\hline Chronic disease & 22 (37.9\%) & $10(19.6 \%)$ & \\
\hline Anxiety or depression & $10(17.2 \%)$ & $13(25.4 \%)$ & \\
\hline HIVIAIDS & $4(6.9 \%)$ & $2(3.9 \%)$ & \\
\hline
\end{tabular}

Notes: Continuous variables were presented as means $\pm S D$ and compared using Student's $t$-test; Dichotomous variables were presented as $\mathrm{n}, \%$ and compared using Chi-squared test.

belief in using amphetamine to quit methadone or fight against side effects of drugs such as heroin. The main ways of abuse were oral and nasal inhalation. Injection was relatively rare. $55.0 \%$ of the patients used drugs at their home (Table 2).

\section{Comparison of ASI and ATS}

Mean ASI score of drug abuse in the MMT group was higher than the BMT group $((0.28 \pm 0.07)$ vs $(0.22 \pm 0.08)$; $P=0.041)$. However, there were no significant differences in physical health status, occupational/social support status, alcohol abuse, family/social relationship, and criminal and mental status (all $P>0.05$ ) (Table 3).

\section{Comparison of Impulsiveness}

Total and subscale scores of Impulsiveness were all higher in the MMT group than those in the MMT control group. 
Table 2 ATS Use Characteristics

\begin{tabular}{|c|c|c|c|}
\hline Characteristics & MMT $(n=58)$ & BMT $(n=51)$ & $P$-value \\
\hline Drug use patterns (n,\%) & & & 0.492 \\
\hline Sniffing & $28(48.3 \%)$ & 20 (39.2\%) & \\
\hline Oral & $24(4 \mathrm{I} .4 \%)$ & 27 (52.9\%) & \\
\hline Injection & $6(10.3 \%)$ & $4(7.8 \%)$ & \\
\hline The reason for the first use of ATS $(n, \%)$ & & & 0.997 \\
\hline Curiosity & $15(25.9 \%)$ & $13(25.5 \%)$ & \\
\hline Influenced by friends & $16(27.6 \%)$ & 14 (27.5\%) & \\
\hline Improve sexual function & $7(12.1 \%)$ & $7(13.7 \%)$ & \\
\hline Improve depressive symptoms & $8(13.8 \%)$ & $8(15.7 \%)$ & \\
\hline Withdrawal of opioid & $6(10.3 \%)$ & $5(9.8 \%)$ & \\
\hline Cheaper price & $6(10.3 \%)$ & $4(7.8 \%)$ & \\
\hline Place of abuse $(n, \%)$ & & & 0.792 \\
\hline Own home & $33(56.9 \%)$ & 27 (52.9\%) & \\
\hline Friends' home & $17(29.3 \%)$ & $18(35.3 \%)$ & \\
\hline Entertainment venues & $8(13.8 \%)$ & $6(11.8 \%)$ & \\
\hline The source of drug $(n, \%)$ & & & 0.547 \\
\hline Friends & $19(32.8 \%)$ & 14 (27.5\%) & \\
\hline Black market & 39 (67.2\%) & 37 (72.5\%) & \\
\hline Interval of ATS use (days) & $5.53 \pm 3.99$ & $9.15 \pm 5.51$ & $0.039 *$ \\
\hline Duration for ATS (months) & $21.79 \pm 13.45$ & $20.77 \pm 13.33$ & 0.834 \\
\hline Duration in maintain (months) & $50.53 \pm 22.48$ & $48.92 \pm 21.61$ & 0.842 \\
\hline Age of initial opioid (years) & $23.84 \pm 4.94$ & $24.30 \pm 5.12$ & 0.798 \\
\hline Opioid use duration before MMT or BMT (years) & $7.42 \pm 6.41$ & $3.34 \pm 3.53$ & $0.046^{*}$ \\
\hline Opioid dose before MMT or BMT (g) & $1.68 \pm 0.67$ & $1.17 \pm 0.68$ & $0.04 I^{*}$ \\
\hline Used opioid in last 30 days $(n, \%)$ & $16(27.6 \%)$ & $10(19.6 \%)$ & 0.837 \\
\hline
\end{tabular}

Notes: Continuous variables are presented as means \pm SD and compared using Student's $t$-test; Dichotomous variables were presented as n, \% and compared using Chisquared test. $* P<0.05:$ MMT group vs BMT group.

The patients in the BMT group had significantly higher total and subscale scores of unplanned impulsiveness and motor impulsiveness than those in the BMT control group (total score: $(80.39 \pm 12.04)$ vs $(70.76 \pm 10.54)$; Nonplanning: (27.57 \pm 7.59$)$ vs (23.04 \pm 6.85$)$; Motor: (31.09 $\pm 8.06)$ vs $(26.09 \pm 7.27))$. However, there was no difference in Attentional Impulsiveness. Similarly, MMT and BMT did not differ in either total or three subscale scores (all $P>0.05)$ (Table 4).

\section{Discussion}

In this study, we found that the impulsivity of the patients with simple MMT or BMT was higher than that in the healthy controls. The OMT patients with ATS abuse had higher impulsivity than those without ATS abuse. In addition, the frequency and severity of ATS use in the MMT group was higher than that in the BMT group.
It has been reported that buprenorphine has less physical dependence, lower severity and shorter duration of withdrawal symptoms than methadone. ${ }^{7}$ Furthermore, buprenorphine is also more likely to be discontinued, which make it superior to methadone in terms of drug safety and potential for abuse. ${ }^{35}$ However, buprenorphine is a semi-agonist of opioid receptor and its effect of controlling heroin withdrawal symptoms is not as good as that of methadone. ${ }^{23,36}$ It probably is one of the reasons why some opioid addicts have to choose MMT to control heroin withdrawal symptoms. Patients with high daily drug dose usually chose MMT for detoxification, which may suggest MMT is more suitable for severe opioid addicts with long duration and high dose in comparison with BMT. It has also been reported that patients who received BMT were generally less severely addicted. ${ }^{37}$ Therefore, as former opioid addicts, patients receiving 
Table 3 Addiction Severity Index Scores

\begin{tabular}{|l|l|l|l|}
\hline & MMT (n=58) & BMT (n=5 I) & $P$-value \\
\hline Medical & $0.20 \pm 0.08$ & $0.19 \pm 0.1 I$ & 0.776 \\
Employment & $0.7 I \pm 0.1 I$ & $0.67 \pm 0.17$ & 0.435 \\
Drug & $0.28 \pm 0.07$ & $0.22 \pm 0.08$ & $0.04 I^{*}$ \\
Alcohol & $0.07 \pm 0.08$ & $0.08 \pm 0.06$ & 0.746 \\
Family/Social & $0.16 \pm 0.07$ & $0.17 \pm 0.09$ & 0.648 \\
Legal & $0.12 \pm 0.04$ & $0.13 \pm 0.08$ & 0.841 \\
Psychological & $0.23 \pm 0.08$ & $0.20 \pm 0.12$ & 0.567 \\
\hline
\end{tabular}

Notes: Continuous variables are presented as means \pm SD and compared using Student's $t$-test; ${ }^{*} p<0.05$ : MMT group vs BMT group.

MMT and BMT have different degrees of opioid addiction before drug maintenance therapy. For patients in the MMT group, it is likely that long-term, larger dose use of opioids lead to greater tolerance of the drug, and hence more severe abuse of methamphetamine.

Impulsivity is a multidimensional neural psychological structure, which is simply defined as the tendency to act prematurely without foresight. ${ }^{25,26,38,39}$ At present, most studies on the impulsivity of addicts have focused on cocaine abusers. ${ }^{27,28,40,41}$ However, there are few studies on the impulsivity of patients under drug maintenance therapy who simultaneously abuse stimulants. Previous studies have showed that the impulsivity of cocaine addicts is significantly higher than that of healthy controls. $^{27,40}$ The impulsivity of MMT patients simultaneously using cocaine was also higher than that of normal controls. $^{29,42}$ ATS use may also exert an impact on impulsivity scores, as has been demonstrated by many former studies. Thus, impulsivity might also be influenced in its level by stimulant administration.

In China, the abuse of cocaine is relatively rare, while the abuse of methamphetamine stimulants is more common. ${ }^{43}$ As a result, patients under MMT are relatively more likely to be using methamphetamine stimulants. ${ }^{30,32,44,45}$ In some provinces, the rate of methamphetamine abuse among MMT outpatient patients has even exceeded $50 \%{ }^{46}$ In this study, we found the impulsivity of MMT and BMT users who were using ATS in combination was higher than that of the normal non-abusers, which is in accordance with previous reports. $^{28,41}$ However, we also found that the MMT and BMT patients who were using ATS in combination had higher impulsivity than those without ATS. This finding is different from the study of Nielsen et al., in which no significance is found between ATS-abusing and non-ATSabusing OMT patients. ${ }^{24,25,42}$ The discrepancy may be attributable to the different duration of maintenance treatment between MMT and BMT populations. The length of maintenance treatment in our study was shorter than the study of Nielsen et al. Patients with lower impulsivity tend to stay in the treatment longer than those with higher impulsivity. Moreover, our study had limited sample size. As a result, patients with higher impulsivity were inadvertently selected by our study. In addition, our results suggested that, as former opioid addicts, there was no significant difference in impulsivity between MMT and BMT for those who had combined ATS.

There are also several limitations in this study. First, this study had limited sample size and the duration of ATS abuse in our participants was all relatively short. There may be some differences in the experimental results compared with those with longer ATS abuse time. Secondly, due to the high loss rate of drug maintenance in clinics at present, the duration of maintenance treatment in our study was also relatively short. Whether this will affect the results is also unknown. Finally, our study lacks the validation of the Amphetamine Abuse Questionnaire (AAQ).

\section{Conclusion}

The drug maintenance treatment in patients abusing amphetamines had a greater impulsiveness than those having other simple maintenance treatments. Patients under MMT may be more addicted to amphetamines in comparison with those having BMT, which is probably due to the characteristics of long-term drug abuse. Tailored interventions and training methods should be developed to reduce

Table 4 Barratt Impulsiveness Scale (Means \pm SD)

\begin{tabular}{|l|l|l|l|l|}
\hline Group & Total Score & Attentional & Non-planning & Motor \\
\hline MMT $(n=58)$ & $80.05 \pm 12.93^{*}$ & $23.15 \pm 5.63^{*}$ & $27.53 \pm 6.44^{*}$ & $30.13 \pm 6.26^{*}$ \\
MMT control $(n=40)$ & $69.04 \pm 8.42$ & $20.23 \pm 4.65$ & $22.61 \pm 5.26$ & $26.19 \pm 7.18$ \\
BMT $(n=5 I)$ & $80.39 \pm 12.04^{\#}$ & $21.72 \pm 6.45$ & $27.57 \pm 7.59^{\#}$ & $31.09 \pm 8.06^{\#}$ \\
BMT control $(n=40)$ & $70.76 \pm 10.54$ & $21.61 \pm 6.31$ & $23.04 \pm 6.85$ & $26.09 \pm 7.27$ \\
\hline
\end{tabular}

Notes: Continuous variables are presented as means \pm SD and compared using Student's $t$-test; $* P<0.05$ : MMT group vs MMT control group. ${ }^{\#} P<0.05$ : BMT group vs BMT control group. 
impulsiveness and improve self-control of patients under drug maintenance treatment, especially MMT.

\section{Funding}

This research was supported by the National Key R\&D Program of China (No. 2017YFC1310400), Natural Science Foundation of China (No.82071499) and the Ningbo Science and Technology Planning Program (NO. 2019A610296).

\section{Disclosure}

The authors declare that they have no conflicts of interest.

\section{References}

1. Dowell D, Arias E, Kochanek K, et al. Contribution of opioidinvolved poisoning to the change in life expectancy in the United States 2000-2015. JAMA. 2017;318(11):1065-1067. doi:10.1001/ jama.2017.9308

2. Tsui JI, Burt R, Thiede H, et al. Utilization of buprenorphine and methadone among opioid users who inject drugs. Subst Abus. 2018;39(1):83-88. doi:10.1080/08897077.2017.1363844

3. Mintzer MZ, Stitzer ML. Cognitive impairment in methadone maintenance patients. Drug Alcohol Depend. 2002;67(1):41-51. doi:10.1016/S0376-8716(02)00013-3

4. Nosyk B, Fischer B, Sun H, et al. High levels of opioid analgesic co-prescription among methadone maintenance treatment clients in British Columbia, Canada: results from a population-level retrospective cohort study. Am J Addict. 2014;23(3):257-264. doi:10.1111/ j.1521-0391.2014.12091.x

5. Kapitány-Fövény M, Farkas J, Pataki PA, et al. Novel psychoactive substance use among treatment-seeking opiate users: the role of life events and psychiatric symptoms. Hum Psychopharmacol. 2017;32 (3):e2602. doi:10.1002/hup.2602

6. Yin W, Hao Y, Sun X, et al. Scaling up the national methadone maintenance treatment program in China: achievements and challenges. Int $J$ Epidemiol. 2010;39(Suppl 2):ii29-ii37. doi:10.1093/ije/dyq210

7. Schottenfeld RS, Chawarski MC, Mazlan M. Maintenance treatment with buprenorphine and naltrexone for heroin dependence in Malaysia: a randomized, double-blind, placebo-controlled trial. Lancet. 2008;371(9631):2192-2200.

8. Milward J, Lynskey M, Strang J. Solving the problem of non-attendance in substance abuse services. Drug and Alcohol Rev 2014;33(6):625-636. doi:10.1111/dar.12194

9. Hser YI, Li J, Jiang H, et al. Effects of a randomized contingency management intervention on opiate abstinence and retention in methadone maintenance treatment in China. Addiction. 2011;106 (10):1801-1809. doi:10.1111/j.1360-0443.2011.03490.x

10. Umbricht A, Defulio A, Winstanley EL, et al. Topiramate for cocaine dependence during methadone maintenance treatment: a randomized controlled Trial. Drug Alcohol Depend. 2014;140:92-100. doi:10.1016/j.drugalcdep.2014.03.033

11. United Nations Office on Drugs and Crime. World Drug Report Volume 1. Analysis. Vienna: United Nations Office on Drugs and Crime; 2007.

12. Koester P, Tittgemeyer M, Wagner D, Becker B, GouzoulisMayfrank E, Daumann J. Cortical thinning in amphetamine-type stimulant users. Neuroscience. 2012;221:182-192. doi:10.1016/j. neuroscience.2012.06.049
13. Vigezzi P, Gugliemino L, Marzorati P. Multimodal drug addiction treatment: a field comparison of methadone and buprenorphine among heroin-and cocaine -dependent patients. $J$ Subst Abuse Treat. 2006;31(1):3-7. doi:10.1016/j.jsat.2006.03.007

14. DeMaria PA Jr, Sterling R, Weinstein SP. The effect of stimulant and sedative use on treatment outcome of patients admitted to methadone maintenance treatment. Am $J$ Addict. 2000;9(2):145-153. doi:10.1080/10550490050173217

15. Jianhua L, Xinyue L. Current status of drug use and HIV/AIDS prevention in drug users in China. J Food Drug Anal. 2013;21(4): S37-41. doi:10.1016/j.jfda.2013.09.031

16. Ornstein TJ, Iddon JL, Baldacchino AM, et al. Profiles of cognitive dysfunction in chronic amphetamine and heroin abusers. Neuropsychopharmacology. 2000;23(2):113-126. doi:10.1016/ S0893-133X(00)00097-X

17. Cheng Z, Chen GH, Dai MM, et al. New psychoactive substances abuse among patients with access to methadone maintenance treatment in jiangsu province: a case-control Study. Chin J Epidemiol. 2018;39(5):625-630.

18. Li JH, Li XY. Current status of drug use and HIV/AIDS prevention in drug users in China. J Food Drug Anal. 2013;21(4):S37-S41.

19. Belin D, Mar AC, Dalley JW, et al. High impulsivity predicts the switch to compulsive cocaine taking. Science. 2008;320 (5881):1352-1355. doi:10.1126/science.1158136

20. Kathryn A, Anastasia CN. Serotonin at the Nexus of impulsive and cue reactivity in cocaine addiction. Neuropharmacology. 2014;76 (00):460-478. PMID: 23850573.

21. Nigg JT, Wong MM, Martel MM, et al. Poor response inhibition as a predictor of problem drinking and illicit drug use in adolescents at risk for alcoholism and other substance use disorders. $J$ Am Acad Child Adolesc Psychiatry. 2006;45(4):468-475. [PMID:16601652]. doi:10.1097/01.chi.0000199028.76452.a9

22. Perry JL, Carroll ME. The role of impulsive behavior in drug abuse. Psychopharmacology. 2008;200(1):1-26.

23. Reynolds B. A review of delay-discounting research with humans: relations to drug use and gambling. Behav Pharmacol. 2006;17 (8):651-667. doi:10.1097/FBP.0b013e3280115f99

24. Bickel WK, Jarmolowicz DP, Mueller ET, et al. Excessive discounting of delayed reinforcers as a trans-disease process contributing to addiction and other disease-related vulnerabilities: emerging evidence. Pharmacol Ther. 2012;134(3):287-297. doi:10.1016/j.pharmthera.2012.02.004

25. Yaghubi M, Zargar F, Akbari H. Comparing effectiveness of mindfulness-based relapse prevention with treatment as usual on impulsivity and relapse for methadone-treated patients: a randomized clinical Trial. Addict Health. 2017;9(3):156-165.

26. Fernández-Serrano MJ, Perales JC, Moreno-López L, et al. Neuropsychological profiling of impulsivity and compulsivity in cocaine dependent individuals. Psychopharmacology. 2012;219 (2):673-683. doi:10.1007/s00213-011-2485-z

27. Stevens L, Roeyers H, Dom G, et al. Impulsivity in cocaine-dependent individuals with and without attention-deficit/hyperactivity disorder. Eur Addict Res. 2015;21(3):131-143. doi:10.1159/000369008

28. Moeller FG, Barratt ES, Dougherty DM, et al. Psychiatric aspects of impulsivity. Am J Psychiatry. 2001;158(11):1783-1793. doi:10.1176/ appi.ajp.158.11.1783

29. Moeller FG, Dougherty DM, Barratt ES, et al. The impact of impulsivity on cocaine use and retention in treatment. J Subst Abuse Treat. 2001;21(4):193-198. doi:10.1016/S0740-5472(01)00202-1

30. McLellan AT, Luborsky L, Cacciola J, et al. New data from the Addiction Severity Index: reliability and validity in three cen-ters. $J$ Nerv Ment Dis. 1985;173(7):412-423. doi:10.1097/00005053198507000-00005

31. Liang T, Liu EW, Zhong H. Reliability and validity of addiction severity index(ASI) Chinese version in evaluation of heroin addiction methadone maintenance treatment. Chin J Drug Depend. 2007;16 (6):451-458. 
32. Patton JH, Stanford MS, Barratt ES. Factor structure of the barratt impulsiveness scale. J Clin Psychol. 1995;51(6):768-774. doi:10.1002/1097-4679(199511)51:6<768::AIDJCLP2270510607>3.0.CO;2-1

33. Zhou L, Xiao SY, He XY. Reliability and validity of chinese version of barratt impulsiveness scale-11. Chin J Clin Psychol. 2006;14 (4):343-344.

34. Haden SC, Shiva A. Trait impulsivity in a forensic inpatient sample: an evaluation of the Barratt impulsiveness scale. Behav Sci Law. 2008;26(6):675-690. doi:10.1002/bsl.820

35. Bishop B, Gilmour J, Deering D. Readiness and Recovery: transferring between methadone and buprenorphine/naloxone for the treatment of opioid use disorder. Int J Ment Health Nurs. 2019;28 (1):226-236. doi:10.1111/inm.12523

36. Kahan M, Srivastava A, Ordean A. Buprenorphine: new treatment of opioid addiction in primary care. Can Fam Physician. 2011;57 (3):281-289.

37. Srivastava A, Kahan M, Nader M. Primary care management of opioid use disorders: abstinence, methadone, or buprenorphine-naloxon? Can Fam Physician. 2017;63(3):200-205.

38. Dalley JW, Everitt BJ, Robbins TW. Impulsivity, compulsivity, and top-down cognitive control. Neuron. 2011;69(4):680-694. doi:10.1016/j.neuron.2011.01.020

39. Evenden JL. Varieties of impulsivity. Psychopharmacology. 1999;146 (4):348-361. doi:10.1007/PL00005481

40. Ross EL, Yoon JH, Mahoney JJ, et al. The impact of self-reported life stress on current impulsivity in cocaine dependent adults. Prog Neuropsychopharmacol Biol Psychiatry. 2013;46:113-119. doi:10.1016/j.pnpbp.2013.06.002
41. Pike E, Marks KR, Stoops WW, et al. Cocaine -related stimuli Impair inhibitory control in cocaine users following short stimulus onset asynchronies. Addiction. 2015;110(8):1281-1286. doi:10.1111/ add. 12947

42. Nielsen DA, Ho A, Bahl A, et al. Former heroin addicts with or without a history of cocaine dependence are more impulsive than controls. Drug Alcohol Depend. 2012;124(1-2):113-120. doi:10.1016/j.drugalcdep.2011.12.022

43. Jia Z-J, Yan S-Y, Bao Y-P, Lian Z, Zhang H-R, Liu Z-M. Sexual behavior differences between amphetamine-type stimulants users and heroin users. J Addict Med. 2013;7(6):422-427. doi:10.1097/ ADM.0b013e3182a952b2

44. Wang R, Ding Y, Bai H, et al. Illicit heroin and methamphetamine use among methadone maintenance treatment patients in Dehong Prefecture of Yunnan Province, China. PLoS One. 2015;10(7): e0133431. doi:10.1371/journal.pone.0133431

45. Du J, Sun H, Huang D, et al. Use trajectories of Amphetamine-type stimulants(ATS) in Shanghhai, China. Drug Alcohol Depend. 2014;143:44-50. doi:10.1016/j.drugalcdep.2014.06.031

46. Cheng Z, Dai MM, Cao XB. [Current situation on new psychoactive substances abuse among methadone maintenance treatment patients in China]. Zhonghua Liu Xing Bing Xue Za Zhi. 2018;39(4):536-540. [Chinese]. doi:10.3760/cma.j.issn.0254-6450.2018.01.030

\section{Publish your work in this journal}

Drug Design, Development and Therapy is an international, peerreviewed open-access journal that spans the spectrum of drug design and development through to clinical applications. Clinical outcomes, patient safety, and programs for the development and effective, safe, and sustained use of medicines are a feature of the journal, which has also been accepted for indexing on PubMed Central. The manuscript management system is completely online and includes a very quick and fair peer-review system, which is all easy to use. Visit http://www. dovepress.com/testimonials.php to read real quotes from published authors. 